\title{
Thema der nächsten Ausgabe:
}

\section{Hybride Wertschöpfung}

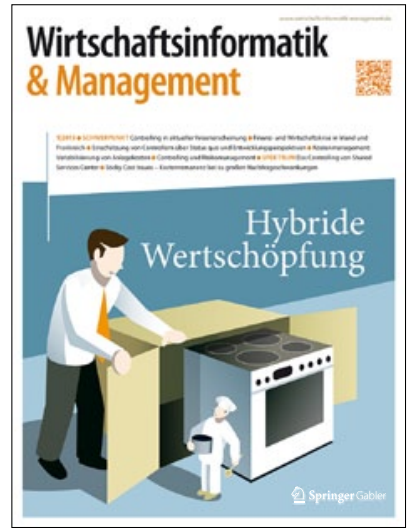

Hybride Wertschöpfung ist Alltag in deutschen Unternehmen. Trotzdem ist der Begriff weit davon entfernt, allgemein bekannt zu sein. Die meisten Firmen verkaufen heute neben den Produkten, die sie herstellen, zusätzliche Dienstleistungen. Das Spektrum reicht dabei vom klassischen Wartungsvertrag bis hin zur Unternehmensberatung. Begleitet wird beispielsweise der gesamte Lebenszyklus einer Maschine, von der Planung über die Inbetriebnahme bis hin zum Recycling am Ende der Produktlebensdauer. Sinnvoll sind diese zusätzlichen Angebote schon deshalb, weil gerade Investitionsgüter nur sehr selten gekauft und dann über Jahrzehnte verwendet werden. Hybride Wertschöpfung sorgt für gleichmäßigeren Umsatz.

\section{Impressum}

Wirtschaftsinformatik \& Management Die Praktikerzeitschrift für IT-Entscheider www.wirtschaftsinformatik-

management.de

Ausgabe 5 | 2014 |06. Jahrgang

ISSN 1867-5905

Verlag

Springer Gabler

Springer Fachmedien Wiesbaden GmbH

Abraham-Lincoln-Straße 46

65189 Wiesbaden

www.springer-gabler.de

Amtsgericht Wiesbaden|HRB 9754

USt-IdNr. DE811148419

Geschäftsführer

Armin Gross, Peter Hendriks,

Joachim Krieger.

Gesamtleitung Anzeigen und Märkte Armin Gross

Gesamtleitung Produktion

Olga Chiarcos

Gesamtleitung Publishing

Stefanie Burgmaier

Advisory Board

Dr. Udo Bub,

Deutsche Telekom Laboratories

Prof. Dr. Dieter Ehrenberg,

Universität Leipzig

Prof. Dr. Manfred Grauer,

Universität Siegen

Dr. Jürgen Laartz,

McKinsey \& Company, Inc.

Prof. Dr. Dr. h.c. mult. Peter Mertens,
Universität Erlangen-Nürnberg Prof. Dr. Matthias Schumann Universität Göttingen

Prof. Dr. Susanne Strahringer,

TU Dresden

Prof. Dr. Dr. h.c. Norbert Szyperski,

Universität Köln

Prof. Dr. Udo Winand,

Universität Kassel

Dr. Peter Zencke,

SAP Deutschland AG \& C. KG

Chefredakteur

Peter Pagel M. A.

Tel.: +49 (0)611 7878-329

peter.pagel@springer.com

Kundenservice

Springer Customer Service GmbH

Springer Gabler-Service

Haberstr. 7|D-69126 Heidelberg

Telefon: +49(0)6221 345-4303

Fax: +49 (0)6221 345-4229

Montag - Freitag 8:00 Uhr - 18:00 Uhr

springergabler-service@springer.com

Nur für Mitglieder der Gesellschaft für Infor-

matik (GI): Andrea Schlaus

Tel.: +49 (0)228 302-153

andrea.schlaus@gi-ev.de

Produktmanagement

Jens Fischer

Tel.: +49 (0)611 7878-340

jens.fischer@springer.com

Verkaufsleitung Anzeigen

Carolin Habermann

Tel.: +49 (0)611 7878-211 carolin.habermann@springer.com Anzeigenpreise: Es gelten die Mediainformationen vom 01.01.2012

Anzeigendisposition

Michaela Friedt

Tel.: +49 (0)611 7878-618

michaela.friedt@springer.com

Layout und Produktion

Erik Dietrich

Tel.: +49 (0)611 7878-170

erik.dietrich@springer.com

Titelbild

Jörg Block

Partner

Allianz Managed Operations \& Services SE, Commerzbank AG, McKinsey \& Company Inc., SAP Deutschland AG \& Co. KG, Deutsche Telekom AG

Bezugsmöglichkeit

Bestellen Sie Ihr Heftabo unter: www.meinfachwissen.de/wum

Das Heft erscheint sechsmal jährlich.

Bezugspreise (inkl. MwSt. und Versand): Privatabonnement 119,00 EUR; Unternehmensabonnement 149,00 EUR; Studentenabonnement 77,00 EUR.

Jedes Abonnement beinhaltet eine Freischaltung für das Online-Archiv. Der Zugang gilt ausschließlich für den einzelnen Empfänger des Abonnements. Für eine Freischaltung des Unternehmens/Bibliothek/ Institution wenden Sie sich bitte an Rüdiger Schwenk: Tel. +49(0)611-7878 357 | ruediger.schwenk@springer.com.
Das Abonnement kann jederzeit zur nächsten erreichbaren Ausgabe schriftlich mi Nennung der Kundennummer gekündigt werden. Eine schriftliche Bestätigung erfolgt nicht. Zuviel gezahlte Beträge für nicht gelieferte Ausgaben werden zurückerstattet.

C Persönliche Mitglieder des Fachbereichs 5 "Wirtschaftsinformatik" der Gesellschaft für Informatike.V. haben die Möglichkeit, die Zeitschrift im Rahmen ihrer Mitgliedschaft zu erhalten.

Druck und Verarbeitung Kliemo, Eupen

Die Zeitschrift und alle in ihr enthaltenen einzelnen Beiträge und Abbildungen sind urheberrechtlich geschützt. Jede Verwertung außerhalb der engen Grenzen des Urheberrechtes ist ohne Zustimmung des Verlages unzulässig und strafbar. Das gilt insbesondere für Vervielfältigungen, Übersetzungen, Mikroverfilmungen und die Einspeicherung in elektronischen Systemen. Nachdruckgenehmigung kann die Redaktion erteilen. Für unverlangt eingesandte Beiträge und Rezensionsexemplare wird nicht gehaftet.

Jede im Bereich eines gewerblichen Unternehmens hergestellte oder benützte Kopie dient gewerblichen Zwecken gem. §54 (2) UrhG und verpflichtet zur Gebührenzahlung an die VG WORT, Abteilung Wissenschaft, Goethestr. 49, 80336 München, von der die einzelnen Zahlungsmodalitäten zu erfragen sind.
Alle Rechte vorbehalten. Kein Teil dieser Zeitschrift darf ohne schriftliche Genehmigung des Verlags vervielfältigt oder verbreitet werden. Unter dieses Verbot fällt insbesondere die gewerbliche Vervielfältigung per Kopie, die Aufnahme in elektronische Datenbanken und die Vervielfältigung auf D-Rom und allen anderen elektronischen Datenträgern.

Hinweise für Autoren

Der Autor ist mit der Veröffentlichung seines Beitrags damit einverstanden, dass sein Beitrag außer in der Zeitschrift auch durch Lizenzvergabe in anderen Zeitschriften (auch übersetzt), durch Nachdruck in Sammelbänden (z. B. zu Jubiläen der Zeitschrift oder des Verlags oder in Themenbänden), durch längere Auszüge in Büchern des Verlags auch zu Werbezwecken, durch Vervielfältigung und Verbreitung auf CD-ROM oder anderen Datenträgern, durch Speicherung auf Datenbanken, deren Weitergabe und den Abruf von solchen Datenbanken während der Dauer des Urheberrechtsschutzes an dem Beitrag im In- und Ausland vom Verlag und seinen Lizenznehmern genutzt wird.

(c) Springer Gabler ist Teil von Springer Science+Business Media. 